\title{
Fluctuation theorem for counting statistics in electron transport through quantum junctions
}

\author{
Massimiliano Esposito,* Upendra Harbola, and Shaul Mukamel \\ Department of Chemistry, University of California, Irvine, California 92697, USA
}

(Received 22 September 2006; revised manuscript received 31 January 2007; published 13 April 2007)

\begin{abstract}
We demonstrate that the probability distribution of the net number of electrons passing through a quantum system in a junction obeys a steady-state fluctuation theorem (FT) which can be tested experimentally by the full counting statistics (FCS) of electrons crossing the lead-system interface. The FCS is calculated using a many-body quantum master equation combined with a Liouville space generating function formalism. For a model of two coupled quantum dots, we show that the FT becomes valid for long binning times, and provide an estimate for the finite-time deviations. The Mandel (or Fano) parameter associated with the incoming or outgoing electron transfers shows sub-Poissonian (antibunching) statistics.
\end{abstract}

DOI: 10.1103/PhysRevB.75.155316

PACS number(s): 73.23.Hk, 03.65.Yz, 74.40.+k

\section{INTRODUCTION}

Various far-from-equilibrium relations, such as the Jarzynski relation ${ }^{1-3}$ or the fluctuation theorem (FT),${ }^{4-10} \mathrm{de}-$ rived during the past decade, provide new insights into the emergence of irreversible processes in classical systems. ${ }^{11,12}$ These relations follow from the observation that the ratio of the probability of a system forward and time-reversed trajectory is given by the exponential of a quantity, the trajectory entropy production, which when ensemble averaged, gives the entropy production in the system. These relations quantify the probabilities of observing "nonthermodynamic" trajectories with decreased trajectory entropy production. These probabilities are infinitesimally small in the macroscopic world but are non-negligible in microscopic systems. However, the ensemble averaged dynamics always satisfies the second law (entropy production always grows). With the recent progress in nano and mesoscopic sciences, these probabilities can now be measured. ${ }^{13,14}$ Because in the microscopic world quantum effects can be important, it is interesting to establish whether these fluctuation relations remain true in the quantum regime. This is still an open issue. ${ }^{15-21}$ One of the major obstacles for a general formulation of a quantum FT is the lack of a clear concept of a measurable trajectory. It is therefore helpful to consider systems undergoing a well-defined measurement process. In the counting statistics of photons emitted by an atom or a molecule driven out of equilibrium by a laser field, ${ }^{22-30}$ a trajectory picture is provided by the history of the detected photons. However, the reverse trajectory (where the laser mode absorbs a photon from the molecule) is not easily measurable. Electron counting statistics provides, on the other hand, a clear trajectory picture given by the history of the electron transfers between the system and the leads, where the reverse trajectory (electron moving against the bias) is a measurable quantity. Electron counting statistics in nanosystems has attracted recent interest. ${ }^{31-41}$ Individual electrons crossing quantum dots have been measured. ${ }^{42-45}$ Measuring the statistics of both forward and backward electron transfer events which is essential for verifying the FT has recently been reported in Ref. 46. Most studies have focused on the few lowest moments of the distribution. However, the FT is connected with the probabilities of large fluctuations which re- quire the knowledge of the entire probability distribution.

In this paper, we use the many-electron quantum master equation (QME) derived in Ref. 47 and the generating operator (GO) formalism in Liouville space developed for photon counting statistics ${ }^{22-28}$ to calculate the full counting statistics (FCS) of electrons in biased quantum junctions. Our central formal result is an equation of motion for the GO whose solution can provide the full electron-transfer probability distribution. Neglecting electron-electron interactions, this GO can be factorized into products of single orbital GO, each leading to a statistics similar to the one of the single resonant-level system studied in Ref. 41. By constructing the current GO from the full electron counting GO, we show that the probability distribution of the net number of electrons $k$ entering the system from one of the system-lead interface $\left[k(t)=-\frac{1}{e} \int_{0}^{t} d \tau I(\tau)\right.$, where $I(\tau)$ is the current and $t$ is the measurement time also called binning time], satisfies $P_{t}(k) / P_{t}(-k)=\exp (\beta e V k)$, where $e V$ is the difference between the left and right lead chemical potentials and $\beta=\left(k_{b} T\right)^{-1}$ is the inverse temperature. The probability of observing a current in the direction favored by the bias voltage $V$ is exponentially larger than that of measuring the reverse current. For a nonbiased junction the two currents are equiprobable.

The QME presented in Sec. II is used to calculate the FCS of electrons in Sec. III. In Sec. IV, we define the GO for the net number of electron transfer and show that the FT holds for long measurement times. In Sec. $\mathrm{V}$ we derive closed expressions for the current and its power spectrum and for the Mandel parameter. In Sec. VI, we calculate the probability distribution for the net number of electron transfer for a model of two-coupled quantum dots and analyze the finitetime deviations to the FT. We also study the behavior of the average current and Mandel parameter as a function of the bias and temperature. Conclusions are drawn is Sec. VII.

\section{QUANTUM MASTER EQUATION}

The quantum junction is made of a system (e.g., quantum dot or single molecule) coupled to two leads. The system Hamiltonian reads $H_{S}=\Sigma_{s} \epsilon_{s} c_{s}^{\dagger} c_{s}$, where $c_{s}^{\dagger}\left(c_{s}\right)$ is the Fermi creation (annihilation) operator for the $s$ system orbital. The 
Hamiltonian of the left (right) lead is $H_{L}=\sum_{l} \epsilon_{l} c_{l}^{\dagger} c_{l}$ ( $H_{R}$ $=\Sigma_{r} \epsilon_{r} c_{r}^{\dagger} c_{r}$ ), where $l(r)$ runs over all the left (right) lead orbitals. The entire junction Hamiltonian is $H=H_{S}+H_{L}+H_{R}$ $+H_{T}$ where $H_{T}=\Sigma_{s \nu}\left[T_{s \nu} c_{s}^{\dagger} c_{\nu}+\right.$ H.c. $]$ is the system-leads coupling $(\nu=l, r)$. In Ref. 47, we used second-order perturbation theory in the lead-system interaction and projection operators in the number of electrons in the system to derive a QME describing the dynamics of the reduced system density matrix. The QME can be viewed as a Redfield equation ${ }^{48}$ in Fock space which only contains coherences between states with the same number of electrons. When the relaxation induced by the leads is much slower than the Bohr frequencies of the system, the fast oscillations in the interaction picture can be averaged out. This approximation, known as the rotating-wave approximation (RWA) in quantum optics, is often performed on the Redfield equation to guarantee that the final QME is of the Lindblad form. ${ }^{48-52}$ Our QME finally reads

$$
\begin{aligned}
\dot{\rho}^{n}= & -i\left[H_{S}, \rho^{n}\right]+\sum_{s}\left(v_{s s} c_{s} \rho^{n+1} c_{s}^{\dagger}+w_{s s} c_{s}^{\dagger} \rho^{n-1} c_{s}-v_{s s} c_{s}^{\dagger} c_{s} \rho^{n}\right. \\
& \left.-w_{s s} \rho^{n} c_{s} c_{s}^{\dagger}+\text { H.c. }\right),
\end{aligned}
$$

where $\rho^{n}$ is the reduced density matrix of the system projected into the $n$ electron part of the Fock space. The complete system density matrix is given by $\rho=\Sigma_{n} \rho^{n}$. When summed over $n$, Eq. (1) gives an equation for the total $\rho$ which is of the Lindblad form. ${ }^{47} v_{s s}$ 's and $w_{s s}$ 's are related to lead correlation functions. Assuming a quasicontinuous spectra for the leads, and neglecting the level shift contributions (which only modify the bare Bohr frequencies of the system), we have

$$
v_{s s} \equiv \sum_{y} v_{s s}^{(y)} ; \quad w_{s s} \equiv \sum_{y} w_{s s}^{(y)}
$$

where

$$
\begin{gathered}
v_{s s}^{(y)}=\pi n_{y}\left(\boldsymbol{\epsilon}_{s}\right)\left|T_{s}^{(y)}\left(\boldsymbol{\epsilon}_{s}\right)\right|^{2}\left[1-f_{y}\left(\boldsymbol{\epsilon}_{s}\right)\right], \\
w_{s s}^{(y)}=\pi n_{y}\left(\boldsymbol{\epsilon}_{s}\right)\left|T_{s}^{(y)}\left(\boldsymbol{\epsilon}_{s}\right)\right|^{2} f_{y}\left(\boldsymbol{\epsilon}_{s}\right) .
\end{gathered}
$$

$n_{y}(\epsilon)$ is the density of state of the left or right lead ( $y$ $=L, R)$ at energy $\epsilon \cdot f_{y}(\epsilon) \equiv 1 /\left\{\exp \left[\beta\left(\epsilon-\mu_{y}\right)\right]+1\right\}$ denotes the Fermi distribution of the $y$ lead and $\mu_{y}$ is its chemical potentials. We assume $\mu_{R}=\mu_{0}$ and $\mu_{L}=\mu_{0}+e V$, where $V$ is the bias (see Fig. 1). $w_{s s}^{(y)}$ is the electron transfer rate from lead $y$ to the $s$ orbital and $v_{s s}^{(y)}$ is the rate for the reverse process. These obey the relation

$$
v_{s s}^{(y)}=e^{\beta\left(\epsilon_{s}-\mu_{y}\right)} w_{s s}^{(y)},
$$

so that

$$
\frac{v_{s s}^{(R)} w_{s s}^{(L)}}{v_{s s}^{(L)} w_{s s}^{(R)}}=e^{\beta e V} .
$$

\section{GENERATING FUNCTION FOR ELECTRON- COUNTING STATISTICS}

We consider a system with $M$ orbitals and $n$ spinless electrons, so that $n=0,1, \ldots, M$. The number of $n$-electron
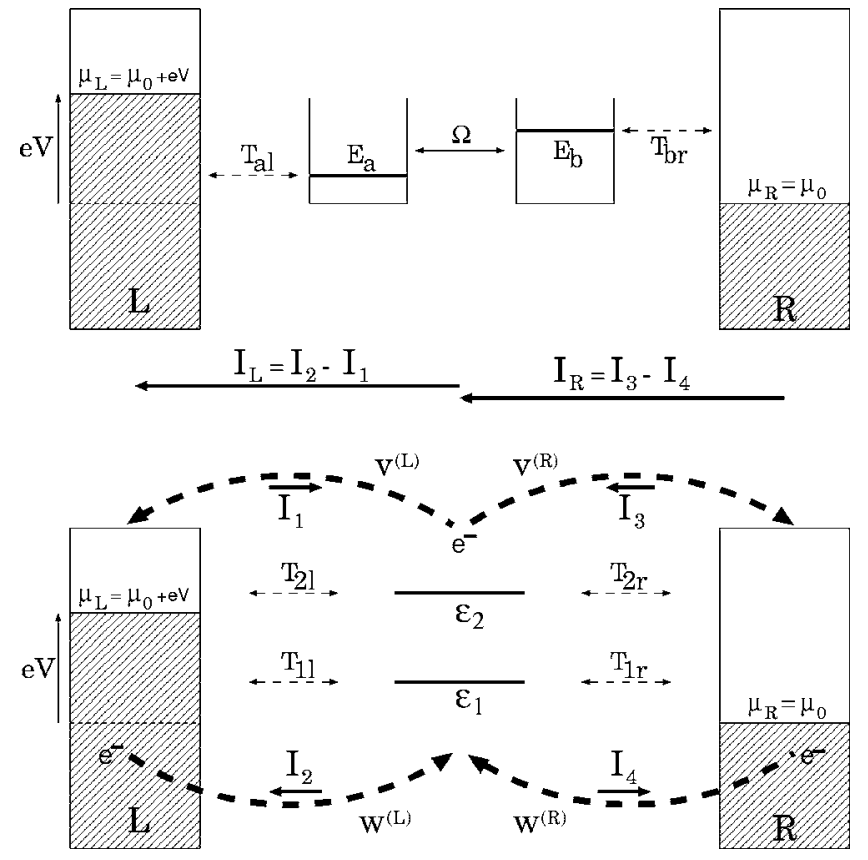

FIG. 1. Schematic representation of the model of two quantum dots $a$ and $b$ coupled in series between two leads used for our numerical results. The upper part depicts the system in the local basis where the Hamiltonian of the dots reads $H_{S}=\Sigma_{i, j} H_{i j} c_{i}^{\dagger} c_{j}$, where $H_{a a}=E_{a}=2, H_{b b}=E_{b}=5$, and $H_{a b}=H_{b a}^{*}=\Omega=1$. The coupling element with the leads are $T_{a l}=0.5, T_{b r}=0.3$, and $T_{a r}=T_{b l}=0$. The lower part depicts the system in the eigenbasis where the Hamiltonian becomes $H_{S}=\Sigma_{s} \epsilon_{s} c_{s}^{\dagger} c_{s}$, with $\epsilon_{1}=1.697$ and $\epsilon_{2}=5.303$. The couplings with the leads become $T_{1 r}=-0.479, T_{2 l}=0.145, T_{1 r}$ $=0.087, T_{2 r}=0.287$. We choose $\mu_{0}=0$ and $n_{y}\left(\epsilon_{s}\right)=\pi^{-1}$ as well as $e=1, \hbar=1$, and $k_{b}=1$, so that the units of energy and temperature is $\Omega$ and the time unit $\Omega^{-1}$. The four possible types of electron transfer (and their associated currents) are represented by the big dashed arrow.

many-body states (hereafter denoted states) is given by $C_{n}^{M}$ $=\frac{M !}{(M-n) ! n !}$. The total number of Fock space states is $N_{\text {tot }}$ $=\sum_{n=0}^{M} C_{n}^{M}=2^{M}$. As a result of the weak lead-system coupling and infinite leads assumption, the Fock space coherences (FSCs) between many-body states with different $n$ are neglected and the number of elements of the full many-body density matrix reduces from $N_{\text {tot }}^{2}=4^{M}$ to $N_{\text {red }}^{2}=\sum_{n=0}^{M}\left(C_{n}^{M}\right)^{2}$. The space of the density matrices where FSCs have been eliminated constitute our reduced Liouville space. By expanding the QME (1) in the eigenbasis of the system, the population dynamics obeys a birth and death master equation which is decoupled from the coherence dynamics. Electrontransfer events are counted by identifying the terms in the QME which are responsible for the transitions between the populations. Their sequence constitutes a "trajectory."

We shall recast the QME (1) in our reduced Liouville space as

$$
|\dot{\rho}\rangle\rangle=(\hat{\mathcal{L}}+\hat{\gamma}+\hat{\Gamma})|\rho\rangle\rangle \equiv \hat{\mathcal{M}}|\rho\rangle\rangle .
$$

$\hat{\mathcal{M}}$ is the generator of the QME. $\hat{\mathcal{L}}$ describes the isolated system dynamics 


$$
\hat{\mathcal{L}}=-i \sum_{s} \epsilon_{s}\left[c_{s}^{\dagger} c_{s}, \cdot\right]
$$

We denote the four possible processes depicted in Fig. 1 by $\eta=1,2,3,4 . \eta=1$ and $\eta=3$ represent electron transfer from the system to the left and the right lead whereas $\eta=2$ and $\eta=4$ represent the electron transfer from the left and the right lead to the system. The orbital through which electron transfer occurs is denoted by $s . \hat{\Gamma}$ is responsible for electron transfers and is made of the nondiagonal terms of the generator which couple the populations

$$
\hat{\Gamma}=\sum_{\nu} \hat{\Gamma}_{\nu}
$$

where $\nu=(\eta, s), \Sigma_{\nu}=\Sigma_{\eta=1}^{4} \Sigma_{s=1}^{M}$, and

$$
\begin{array}{ll}
\hat{\Gamma}_{(1, s)} \equiv 2 v_{s s}^{(L)} c_{s} \cdot c_{s}^{\dagger} ; & \hat{\Gamma}_{(2, s)} \equiv 2 w_{s s}^{(L)} c_{s}^{\dagger} \cdot c_{s}, \\
\hat{\Gamma}_{(3, s)} \equiv 2 v_{s s}^{(R)} c_{s} \cdot c_{s}^{\dagger} ; & \hat{\Gamma}_{(4, s)} \equiv 2 w_{s s}^{(R)} c_{s}^{\dagger} \cdot c_{s} .
\end{array}
$$

$\hat{\gamma}$ describes the diagonal terms of the generator

$$
\hat{\gamma}=\sum_{\nu} \hat{\gamma}_{\nu}
$$

In analogy to $\hat{\Gamma}$, we identify the various contributions to $\hat{\gamma}$ from the different active orbitals

$$
\begin{aligned}
& \hat{\gamma}_{(1, s)} \equiv-v_{s s}^{(L)}\left[c_{s}^{\dagger} c_{s}, \cdot\right]_{+} ; \quad \hat{\gamma}_{(2, s)} \equiv w_{s s}^{(L)}\left[c_{s} c_{s}^{\dagger}, \cdot\right]_{+}, \\
& \hat{\gamma}_{(3, s)} \equiv-v_{s s}^{(R)}\left[c_{s}^{\dagger} c_{s}, \cdot\right]_{+} ; \quad \hat{\gamma}_{(4, s)} \equiv w_{s s}^{(R)}\left[c_{s} c_{s}^{\dagger}, \cdot\right]_{+} .
\end{aligned}
$$

We can now calculate the full electron counting statistics using the formalism developed for photon counting statistics. ${ }^{22-30}$ Starting with a trajectory picture of the QME evolution in terms of electron transfer histories, we shall calculate the probabilities of these trajectories and their associated generating function (GF).

The system density matrix conditional to measuring $\mathbf{k}$ electron transfers during an interval of time $t$ is denoted $\rho^{(\mathbf{k})}(t)$. We use the compact notation defined in Appendix A [see Eq. (A7)]. $\mathbf{k}$ is a vector with components $k_{\nu}$. The probability to measure $\mathbf{k}$ electron transfers during a time interval $t$ is obtained by tracing the conditional system density matrix

$$
P(t, \mathbf{k})=\left\langle\left\langle I \mid \rho^{(\mathbf{k})}(t)\right\rangle\right\rangle .
$$

The trace of a Hilbert space operator $A, \operatorname{Tr} A$, is denoted as a scalar product in Liouville space $\langle\langle I \mid A\rangle\rangle$, where $I$ is the unity operator.

The generating function (GF) associated with this probability distribution is defined as

$$
G(t, \boldsymbol{\lambda}) \equiv \sum_{\mathbf{k}} P(t, \mathbf{k}) e^{\boldsymbol{\lambda} \cdot \mathbf{k}},
$$

where $\boldsymbol{\lambda} \cdot \mathbf{k}=\sum_{\nu} \lambda_{\nu} k_{\nu}$. Similarly, we define the generating operator $(\mathrm{GO})$ as

$$
\left.|G(t, \boldsymbol{\lambda})\rangle\rangle \equiv \sum_{\mathbf{k}}\left|\rho^{(\mathbf{k})}(t)\right\rangle\right\rangle e^{\boldsymbol{\lambda} \cdot \mathbf{k}} .
$$

The GF is obtained by tracing the GO

$$
G(t, \boldsymbol{\lambda})=\langle\langle I \mid G(t, \boldsymbol{\lambda})\rangle\rangle .
$$

An evolution equation for the GO is derived in Appendix A starting with the QME

$$
|\dot{G}(t, \boldsymbol{\lambda})\rangle\rangle=\hat{\mathcal{W}}(\boldsymbol{\lambda})|G(t, \boldsymbol{\lambda})\rangle\rangle,
$$

where

$$
\hat{\mathcal{W}}(\boldsymbol{\lambda})=\hat{\mathcal{M}}+\sum_{\nu}\left(e^{\lambda_{\nu}}-1\right) \hat{\Gamma}_{\nu}
$$

is the generator of the $\mathrm{GO}$ evolution equation. Using the initial condition $|G(0 ; \boldsymbol{\lambda})\rangle\rangle=|\rho(0)\rangle\rangle$, the solution of Eq. (16), given in Appendix B, provides the GF at all times,

$$
G(t, \boldsymbol{\lambda})=\left\langle\left\langle I\left|e^{\hat{\mathcal{W}}(\boldsymbol{\lambda}) t}\right| \rho(0)\right\rangle\right\rangle .
$$

The GF contains the entire information about the electron counting statistics. The probability distribution is obtained by inverting Eq. (13),

$$
P(t, \mathbf{k})=\int_{0}^{2 \pi} d \boldsymbol{\lambda} G(t, i \boldsymbol{\lambda}) e^{-i \boldsymbol{\lambda} \cdot \mathbf{k}} .
$$

Moments of the distribution are given by derivatives of the $\mathrm{GF}$,

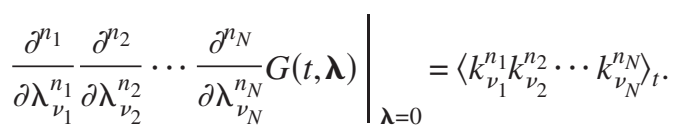

We also define

$$
S(\boldsymbol{\lambda}) \equiv-\lim _{t \rightarrow \infty} \frac{1}{t} \ln G(t, \boldsymbol{\lambda}) .
$$

This will be useful to calculate the statistical properties of steady-state currents.

\section{FLUCTUATION THEOREM FOR THE NET NUMBER OF ELECTRONS TRANSFERRED}

We will now focus on the statistical properties of the charge currents across the junction. We adopt the standard convention that the direction of the charge current is opposite to the electron transfers (see Fig. 1). The number of electrons transferred via process $\eta$ through orbital $s$ during a time interval $t$ is given by

$$
k_{\nu}(t)=-\frac{1}{e} \int_{0}^{t} d \tau I_{\nu}(\tau),
$$

where $I_{\nu}(\tau)$ is the corresponding charge current $[\nu=(\eta, s)]$. The net number of electron transfer events between the left (right) lead-system interface, through orbital $s$, during time $t$ is

$$
k_{(L, s)}(t) \equiv k_{(2, s)}(t)-k_{(1, s)}(t)=-\frac{1}{e} \int_{0}^{t} d \tau I_{(L, s)}(\tau),
$$




$$
k_{(R, s)}(t) \equiv k_{(3, s)}(t)-k_{(4, s)}(t)=-\frac{1}{e} \int_{0}^{t} d \tau I_{(R, s)}(\tau),
$$

where the charge current at the left (right) lead-system interface passing through the $s^{\prime}$ th system orbital is given by $I_{(L, s)}(t) \equiv I_{(2, s)}(t)-I_{(1, s)}(t)\left[I_{(R, s)}(t) \equiv I_{(3, s)}(t)-I_{(4, s)}(t)\right]$. The GF associated with the left and right net number of electron transfer $G\left(t, \boldsymbol{\lambda}_{L}, \boldsymbol{\lambda}_{R}\right)$ is the GF of the FCS $G(t, \boldsymbol{\lambda})$ where $\lambda_{(1, s)}=-\lambda_{(2, s)} \equiv \lambda_{(L, s)}$ and $\lambda_{(4, s)}=-\lambda_{(3, s)} \equiv \lambda_{(R, s)}$. Defining the vectors $\boldsymbol{\lambda}_{L}\left(\boldsymbol{\lambda}_{R}\right)$ with components $\lambda_{(L, s)}\left(\lambda_{(R, s)}\right)$, we have

$$
G\left(t, \boldsymbol{\lambda}_{L}, \boldsymbol{\lambda}_{R}\right)=\sum_{\boldsymbol{\lambda}_{L}, \boldsymbol{\lambda}_{R}} P\left(t, \mathbf{k}_{L}, \mathbf{k}_{R}\right) e^{-\boldsymbol{\lambda}_{L} \cdot \mathbf{k}_{L}} e^{-\boldsymbol{\lambda}_{R} \cdot \mathbf{k}_{R}} .
$$

For clarity, we hereafter consider the left GF. The right one may be calculated similarly. The GF for the left net number of electron transfer $G\left(t, \boldsymbol{\lambda}_{L}\right)$ is defined from Eq. (24) by taking $\boldsymbol{\lambda}_{R}=0$. Using Eq. (B9), we find that the generator $\hat{\mathcal{W}}_{s}\left(\lambda_{(L, s)}\right)$ of the evolution of the single orbital GF $G_{s}\left(t, \lambda_{(L, s)}\right)$ for the net number of electron transfer, via the single orbital $s$, through the left lead-system interface is given by

$$
\begin{aligned}
G_{s}\left(t, \lambda_{(L, s)}\right)= & c_{+}(0) e^{g_{+}\left(\lambda_{(L, s)}\right) t}\left[g_{1 ; 1}^{+}\left(\lambda_{(L, s)}\right)+g_{0 ; 0}^{+}\left(\lambda_{(L, s)}\right)\right] \\
& +c_{-}(0) e^{g_{-}\left(\lambda_{(L, s)}\right) t}\left[g_{1 ; 1}^{-}\left(\lambda_{(L, s)}\right)+g_{0 ; 0}^{-}\left(\lambda_{(L, s)}\right)\right],
\end{aligned}
$$

where, using Eqs. (B8) and (5), the eigenvalues of the generator are given by

$$
g_{ \pm}\left(\lambda_{(L, s)}\right)=-\left(\frac{v_{s s}+w_{s s}}{2}\right) \pm \sqrt{\left(\frac{v_{s s}+w_{s s}}{2}\right)^{2}+v_{s s}^{(R)} w_{s s}^{(L)}\left[e^{-\beta e V}\left(e^{\lambda_{(L, s)}}-1\right)+\left(e^{-\lambda_{(L, s)}}-1\right)\right]} .
$$

These possess the symmetry $g\left(\lambda_{(L, s)}\right)=g\left(\beta e V-\lambda_{(L, s)}\right)$ so that, using $S_{s}\left(\lambda_{(L, s)}\right) \equiv-\lim _{t \rightarrow \infty} \frac{1}{t} \ln G_{s}\left(t, \lambda_{(L, s)}\right)$ and Eq. (25),

$$
S_{s}\left(\lambda_{(L, s)}\right)=S_{s}\left(\beta e V-\lambda_{(L, s)}\right) .
$$

In Appendix B, we show that the many-body GF can be factorized into a product of single orbital GF [see Eq. (B10)]. The GF for the total current (irrespective of the carrying orbitals by setting $\lambda_{(L, s)}=\lambda_{L}$ in $\left.\lambda_{L}\right)$ can therefore be written

$$
G\left(t, \lambda_{L}\right)=\sum_{m}^{M} e^{g_{m}\left(\lambda_{L}\right) t}\left\langle\left\langle I \mid g_{m}\left(\lambda_{L}\right)\right\rangle\right\rangle\left\langle\left\langle\widetilde{g}_{m}\left(\lambda_{L}\right) \mid \rho(0)\right\rangle\right\rangle,
$$

where $\left.g_{m}\left(\lambda_{L}\right),\left|g_{m}\left(\lambda_{L}\right)\right\rangle\right\rangle$ and $\left\langle\left\langle\widetilde{g}_{m}\left(\lambda_{L}\right)\right|\right.$ are, respectively, the many-body eigenvalues, the right and the left eigenvector of the generator. Since the many-body eigenvalues corresponding to populations are made of $2^{M}$ possible sums of singlebody orbital eigenvalues (26), they also satisfy the symmetry $g\left(\lambda_{L}\right)=g\left(\beta e V-\lambda_{L}\right)$ so that, using Eq. (21),

$$
S\left(\lambda_{L}\right)=S\left(\beta e V-\lambda_{L}\right) .
$$

An important point is that the eigenvalues of the generator associated with the right GF are the same as those of the left GF so that $S\left(\lambda_{L}\right)=S\left(\lambda_{R}\right)$. The eigenvectors will, however, be different and $G\left(t, \lambda_{L}\right) \neq G\left(t, \lambda_{R}\right)$. This means that, in general, the electron-transfer statistics at the left and right interface of the junction can be different. However, the statistical properties which can be obtained from $S(\lambda)$ are the same on the two interfaces. These include, for example, the FT [which follows from Eq. (29)], the steady-state average current [see Eq. (D2)], the current power spectrum at zero frequency [see Eq. (D5)], or the asymptotic value of the Mandel parameter [see Eq. (D8)]. Hereafter, we therefore omit the $L, R$ labeling in the corresponding quantities.
In Appendix C, we use the theory of large deviations to show that the symmetry (29) implies at long times

$$
\frac{P(t, k)}{P(t,-k)} \stackrel{t \rightarrow \infty}{=} e^{\beta e V k}
$$

This is the FT for the net number of electrons $k$ crossing the junction at each system-lead interface. Using Eq. (27), we note that the FT also holds for the net number of charges which passed through each orbital $k_{(y, s)}(y=L, R)$. A similar result was pointed out in Ref. 53 for a single resonant level in the large Coulomb repulsion limit excluding double occupancy. The FT implies that measuring electron transfers in the direction favored by the bias is exponentially more probable than the reverse process. The argument of the exponential is proportional to the nonequilibrium constrains of the junction $\beta e V$ so that at equilibrium $(V=0)$ the two probabilities are identical.

\section{AVERAGE CURRENT, MANDEL PARAMETER, AND POWER SPECTRUM}

In Appendix D, we show how currents, moments, and cumulants can be obtained from the GF. Using these results and the expressions for the eigenvalue with the smallest absolute value of the GF generators (B8), we derive closed expressions for the steady-state currents, their zero frequency power spectrum, and the asymptotic value of the Mandel parameter.

Using Eqs. (D2) and (B8), the four steady-state average currents through orbital $s$ are given by

$$
\left\langle I_{(1, s)}\right\rangle_{\mathrm{st}}=-2 e v_{s s}^{(L)} \frac{w_{s s}}{v_{s s}+w_{s s}}, \quad\left\langle I_{(2, s)}\right\rangle_{\mathrm{st}}=-2 e w_{s s}^{(L)} \frac{v_{s s}}{v_{s s}+w_{s s}},
$$




$$
\left\langle I_{(3, s)}\right\rangle_{\mathrm{st}}=-2 e v_{s s}^{(R)} \frac{w_{s s}}{v_{s s}+w_{s s}}, \quad\left\langle I_{(4, s)}\right\rangle_{\mathrm{st}}=-2 e w_{s s}^{(R)} \frac{v_{s s}}{v_{s s}+w_{s s}} .
$$

The total current associated with the process $\eta$ is obtained by summing over the orbitals $\left\langle I_{\eta}\right\rangle_{\mathrm{st}}=\Sigma_{s}^{M}\left\langle I_{(\eta, s)}\right\rangle_{\mathrm{st}}$. The Fano parameter $F_{\nu}(t)$ and the closely related Mandel parameter $M_{\nu}(t)$ of each of these processes $\nu=(\eta, s)$ are defined as

$$
F_{\nu}(t) \equiv M_{\nu}(t)+1 \equiv \frac{\left\langle k_{\nu}^{2}\right\rangle-\left\langle k_{\nu}\right\rangle_{t}^{2}}{\left\langle k_{\nu}\right\rangle_{t}} .
$$

The Mandel parameter vanishes for a Poisson process. $M<0 \quad(M>0)$ implies sub-Poissonian (super-Poissonian) statistics. At steady state, using Eq. (D7), we find

$$
M_{\nu}(\infty)=\frac{1}{e} \frac{\left\langle I_{\nu}\right\rangle_{\mathrm{st}}}{e v_{s s}+w_{s s}} .
$$

Another quantity of interest is the zero-frequency power spectrum of the current $A_{\nu \nu}$ associated with the processes $\nu$ [see Eq. (D5)]. $M_{\nu}(t)$ and $F_{\nu}(t)$ are easily related to it by $A_{\nu \nu}=e\left\langle I_{\nu}\right\rangle_{\mathrm{st}} F_{\nu}(\infty)$. The zero-frequency power spectrum for the total current associated with the process $\eta$ is given by $A_{\eta}=\sum_{s}^{M} A_{\nu v}$. The Mandel or Fano parameters cannot be expressed as such an orbital sum. It is therefore convenient to calculate them from $S_{\eta}$ and $\left\langle I_{\eta}\right\rangle_{\mathrm{st}}$.

Similarly as for the $\eta$ processes, we can use Eq. (26) to calculate the statistical properties of a given junction interface. Using Eq. (D2), the average steady state current via the $s$ orbital reads

$$
\left\langle I_{s}\right\rangle_{s t}=2 e\left(\frac{v_{s s}^{(L)} w_{s s}^{(R)}-v_{s s}^{(R)} w_{s s}^{(L)}}{v_{s s}+w_{s s}}\right) .
$$

Using Eq. (D5), the corresponding zero-frequency power spectrum of the current is given by

$$
A_{s s}=\frac{\left\langle I_{s}\right\rangle_{\mathrm{st}}^{2}}{v_{s s}+w_{s s}}-2 e^{2}\left(\frac{v_{s s}^{(L)} w_{s s}^{(R)}+v_{s s}^{(R)} w_{s s}^{(L)}}{v_{s s}+w_{s s}}\right),
$$

and $A=\sum_{s}^{M} A_{s s}$.

\section{TWO COUPLED QUANTUM DOT MODEL}

We have calculated the probability distribution of the net number of electron transfer at the left lead-system interface for a model of two coupled quantum dots $a$ and $b$. Quantities in local basis will be denoted by a tilde. The Hamiltonian of the dots in the local basis reads

$$
\widetilde{H}_{s}=\left(\begin{array}{cc}
\epsilon_{a} & \Omega \\
\Omega & \epsilon_{b}
\end{array}\right) .
$$

In the orbital eigenbasis, $H_{s}$ is a diagonal matrix with eigenvalues

$$
\epsilon_{1,2}=\frac{\epsilon_{a}+\epsilon_{b}}{2} \pm \sqrt{\left(\frac{\epsilon_{a}-\epsilon_{b}}{2}\right)^{2}+\Omega^{2}} .
$$

The orbitals are labeled $s=1,2$. The two Hamiltonians are connected by a unitary transformation $H_{s}=U \widetilde{H}_{s} U^{\dagger}$, and simi-
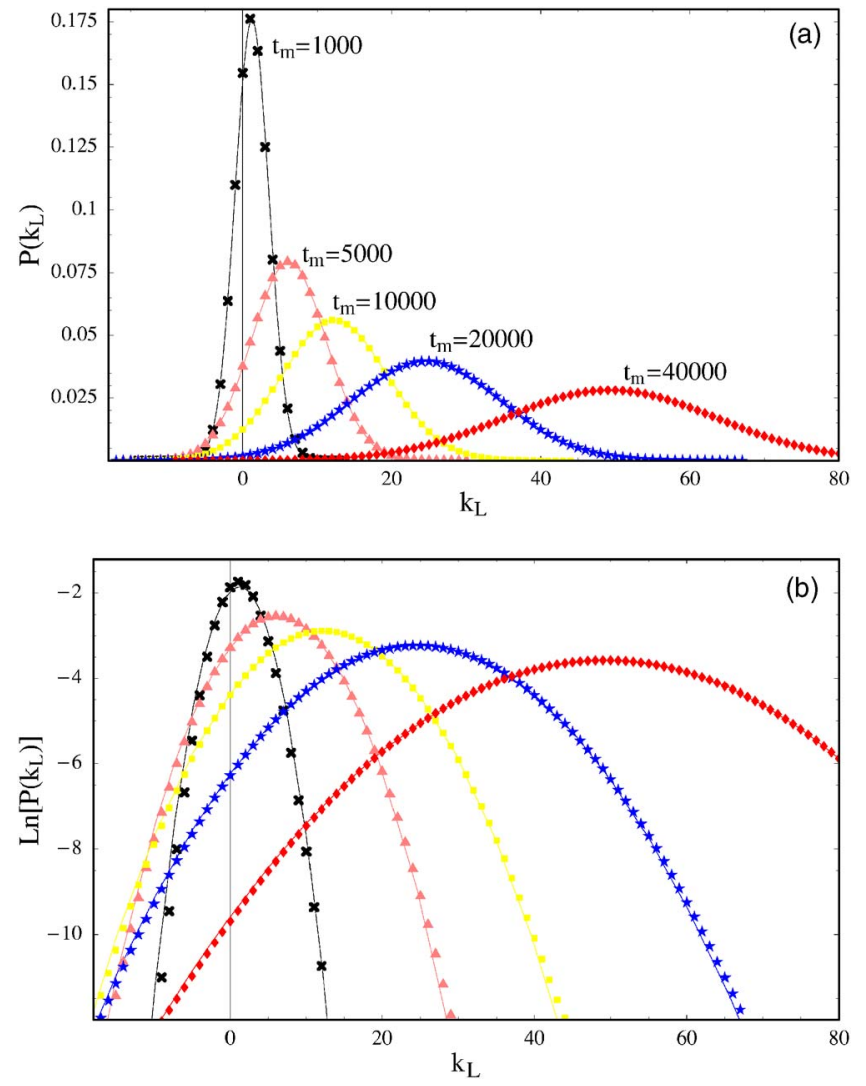

FIG. 2. (Color online) (a) Probability distribution of the net number of electron transfer $k_{L}$ through the left system-lead interface for different measurement times. (b) The logplot highlights the behavior of the tails of the probability distribution. The measurement starts when the junction is at steady state and the different symbols correspond to different measurement times. The solid lines represent Gaussian fits. $e V=0.5$ and $\beta=1$.

larly $\alpha=U \widetilde{\alpha} U^{\dagger}$ and $\beta=U \widetilde{\beta} U^{\dagger}$. We define the couplings between the leads and the dots in the local basis and transform them to the orbital eigenbasis using $U$ (see Fig. 1). Since the two orbitals can be either empty or singly occupied, the system has four many-body states $|00\rangle,|01\rangle,|10\rangle,|11\rangle$. The many-body density matrix in the full Liouville space is thus a vector with 16 elements (4 populations and 12 coherences). In our reduced Liouville space it is a vector with six elements (four populations and two coherences between $|01\rangle$ and $|10\rangle)$. The generator (17) for this model is given in Appendix E.

We have solved the GO equation (16) for the total left current, trace the solution (28) to get the GF, and finally calculate the probability distribution using Eq. (19). We assume that the measurement starts when the junction is at steady state. The parameters used in the numerical simulation are given in the legend of Fig. 1.

In Fig. 2(a), we display the probability distribution of the net number of electrons $k_{L}$ which have crossed the left leadsystem interface for different measurement times and for a fixed temperature and bias. The logarithmic plot in Fig. 2(b) highlights the tails of the distribution. Positive (negative) $k_{L}$ 
represent electrons which move in the direction favored (unfavored) by the bias. As the measurement time increases, the average of the probability distribution moves to the positive direction linearly in time at a speed given by the steady-state current. As expected, the longer the measurement time, the smaller the probability of observing a current flowing against the bias. Since the FT applies in the long time limit, this shows that the FT quantifies the rare fluctuations described by the tails of the probability distribution.

Figure 3(a) shows the logarithm of $P\left(t, k_{L}\right) / P\left(t,-k_{L}\right)$ for different measurement times. The longer the time, the closer the results from the FT. The numerical results suggest that for finite times

$$
\frac{P(t, k)}{P(t,-k)} \approx e^{\left(\beta e V-\alpha_{t}\right) k},
$$

where $\lim _{t \rightarrow \infty} \alpha_{t}=0$. This form seems to be a very good approximation for longer times where the probability to measure at least a few electron transfers becomes significant.

To calculate $\alpha_{t}$, we note that using Eq. (24), Eq. (38) implies that

$$
G\left(t, \lambda_{L}\right) \approx G\left(t, \beta e V-\alpha_{t}-\lambda_{L}\right) .
$$

Since we do not consider very short times, Eq. (28) can be approximated by

$$
\ln G\left(t, \lambda_{L}\right) \approx g_{m_{0}}\left(\lambda_{L}\right) t+B\left(\lambda_{L}\right),
$$

where $m_{0}$ is the index of the eigenvalue with the smallest absolute value and

$$
B\left(\lambda_{L}\right) \equiv \ln \left(\left\langle\left\langle I \mid g_{m_{0}}\left(\lambda_{L}\right)\right\rangle\right\rangle\left\langle\left\langle\widetilde{g}_{m_{0}}\left(\lambda_{L}\right) \mid \rho(0)\right\rangle\right\rangle\right) .
$$

Since $G(t, 0)=G\left(t, \beta e V-\alpha_{t}\right)=1$, we find that

$$
g_{m_{0}}\left(\beta e V-\alpha_{t}\right) t \approx-B\left(\beta e V-\alpha_{t}\right) .
$$

If we consider long enough times for which $\alpha_{t} \ll \beta e V$ and $\alpha_{t} / t \approx 0$, using a first [zero] order expansion of $g_{m_{0}}(\beta e V$ $\left.-\alpha_{t}\right)\left[B\left(\beta e V-\alpha_{t}\right)\right]$ in $\alpha_{e}$ around zero and using Eq. (D2), we get

$$
\alpha_{t} \approx \frac{B(\beta e V)}{e\langle I\rangle_{\mathrm{st}}} \frac{1}{t} .
$$

The average current can be calculated using $\langle I\rangle_{\mathrm{st}}=\Sigma_{s}^{M}\left\langle I_{s}\right\rangle_{\mathrm{st}}$ and Eq. (34). Using $B\left(\lambda_{L}\right)=\sum_{s}^{M} B\left(\lambda_{(L, s)}\right)$, where $B\left(\lambda_{(L, s)}\right)$ $=\ln \left(\left\langle\left\langle I \mid g_{-}\left(\lambda_{(L, s)}\right)\right\rangle\right\rangle\left\langle\left\langle\widetilde{g}_{-}\left(\lambda_{L, s}\right) \mid \rho(0)\right\rangle\right\rangle\right)$ and $\rho(0)$ correspond to steady state, we find that

$$
B(\beta e V)=\sum_{s}^{M} \ln \frac{\left(v_{s s}^{(R)}+w^{(R)}\right)\left(w_{s s}^{2} v_{s s}^{(R)}+v_{s s}^{2} w_{s s}^{(R)}\right)}{v_{s s}^{(R)} w_{s s}^{(R)}\left(w_{s s}+v_{s s}\right)^{2}} .
$$

Notice that since $\exp \left\{B\left(\lambda_{(L, s)}\right)\right\} \geq 1$, then $\alpha_{t} \geq 0$ (the equality only holds when $\beta e V=0$ ).

Figure 3(b) shows that our estimate for $\alpha_{t}$ is in excellent agreement with the values obtained by linearly fitting the results of Fig. 3(a). It should be noted that the results presented on Figs. 2 and 3 correspond to a small bias. For larger bias the probability of the backward processes $P(-k)$ becomes very small which limits the numerical accuracy. How-
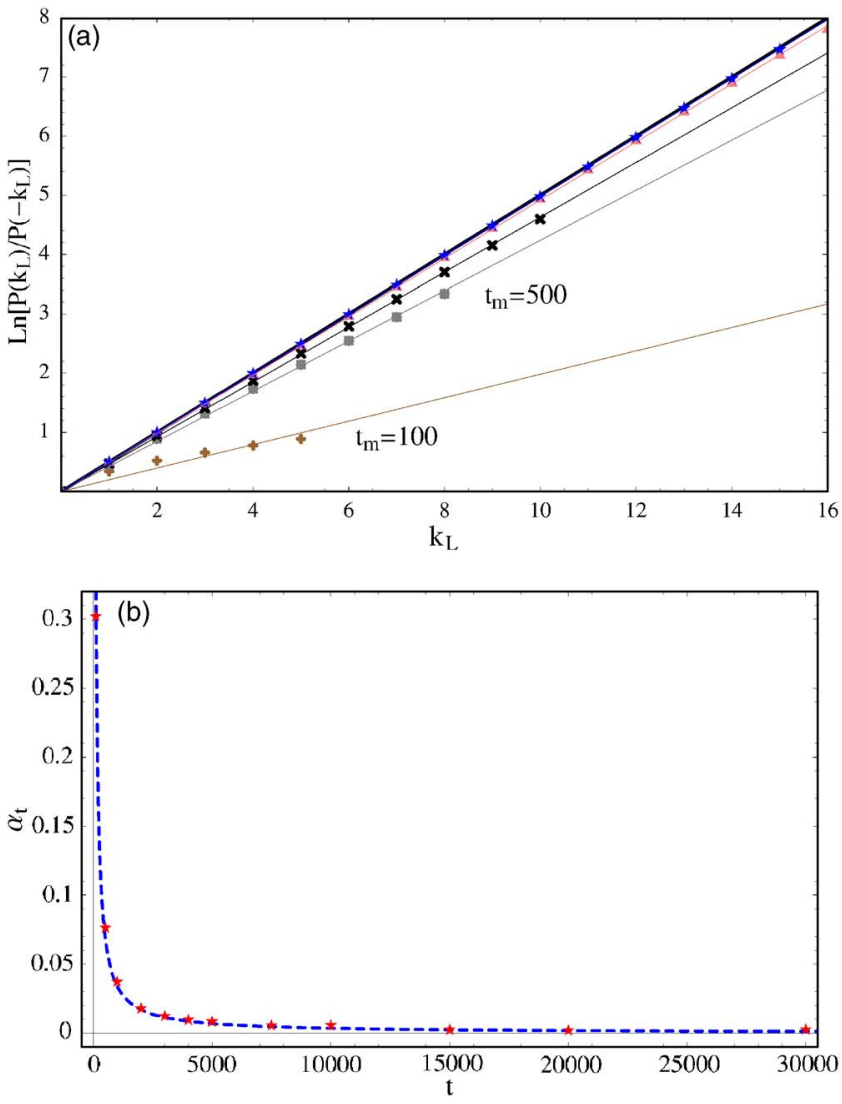

FIG. 3. (Color online) (a) The FT predicts that the logarithm of the ratio of the probability to measure a net number of electron transfer $k_{L}$ in the direction favored by the bias with the probability of measuring the opposite number $-k_{L}$ (which means that a net transfer of $k_{L}$ electron occurred in the direction unfavored by the bias) is given by $\beta e V k_{L}$. This is given by the solid black diagonal line. Different symbols correspond to different measurement times (the symbols not specifically labeled correspond to the same measurement times as in Fig. 2). The FT holds for long measurement times. The solid lines are linear fits. (b) The stars represent the values of $\alpha_{t}$ obtained from the linear fit of the curves from (a) and the dashed line is the approximation for $\alpha_{t}$ given by Eq. (43).

ever, the GF is still numerically accessible for high bias. Figure 4 shows that the GF symmetry (39) on which our method relies is not perfectly preserved for larger bias. It is therefore expected that the accuracy of our method decreases with increasing bias.

One can see in Fig. 2 that the probability distribution can be reasonably well fitted by a Gaussian. Deviations can be observed at very short times or for the tails of the distributions. The GF of a Gaussian probability distribution $P(t, k)$ $=\left(2 \pi \sigma_{t}^{2}\right)^{-1 / 2} \exp \left\{-\left(k-\langle k\rangle_{t}\right)^{2} /\left(2 \sigma_{t}^{2}\right)\right\}$ is given by $G(t, \lambda)$ $=\exp \left\{\lambda^{2} \sigma_{t}^{2} / 2-\lambda\langle k\rangle_{t}\right\}$. The nonzero solution of $G(t, \lambda)=1$ is $\lambda_{0}=2\langle k\rangle_{t} / \sigma_{t}^{2}=2 / F(t)$, where $F(t)$ is the time-dependent Fano parameter associated with the net number of charge transferred through an interface. The GF has the symmetry $G(t, \lambda)=G\left(t, \lambda_{0}-\lambda\right)$. Using the results of Sec. V to calculate $F(\infty)$, we find that for very long measurement times 


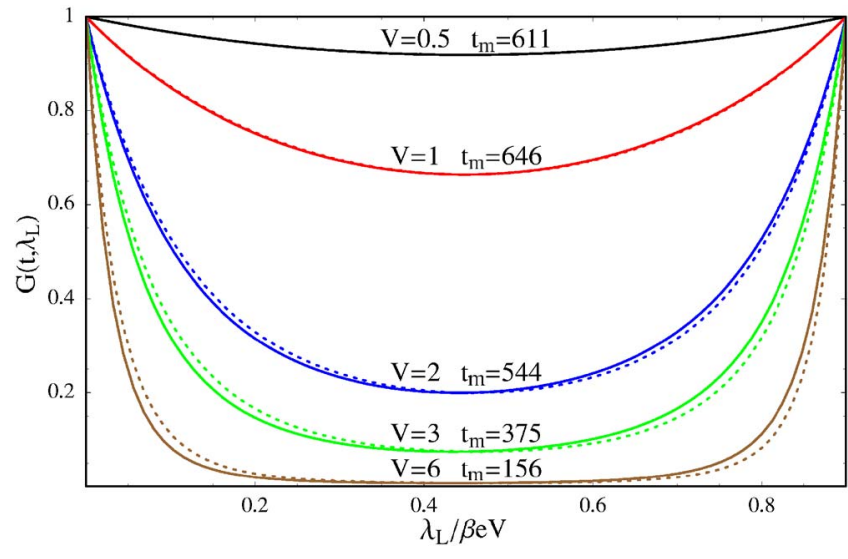

FIG. 4. (Color online) Generating function calculated for different values of the bias and at measurement times $t_{m}$ such that $\alpha_{t_{m}}$ $=0.1 \beta e V . t_{m}$ is calculated by solving $G\left(t_{m}, 0.9 \beta e V\right)=1$. The solid line is $G\left(t_{m}, \lambda_{L}\right)$ and the dotted line is $G\left(t_{m}, 0.9 \beta e V-\lambda_{L}\right)$. The difference between the two curves is a measure of the breakdown of the symmetry (39).

$\lambda_{0}=\beta e V+\mathcal{O}\left(V^{3}\right)$. This indicates that it is only in the low bias limit that the Gaussian approximation can be trusted for describing the tails of the probability distribution which characterize the FT. This was confirmed by numerically studying the GF. We finally notice that our estimate of $\alpha_{t}$ is exact when the Gaussian approximation is satisfied, but can also be applied to non-Gaussian distributions as long as Eqs. (38) and (39) hold.

The average steady-state current associated with the net number of electrons crossing a given junction interface is plotted as a function of the bias in Fig. 5(a). This typical current-voltage characteristic shows that the current increases by steps each time the bias is large enough to make a new orbital contribute to the current (when $e V=\epsilon_{1}=1.697$ or $e V=\epsilon_{2}=5.303$ ). The current associated with the process $\eta$ $=1$ and $\eta=2$ is plotted in Figs. 5(b) and 5(c). The current in Fig. 5(a) is given by the difference between Figs. 5(c) and Fig. 5(b). Temperature, when increased, has the effect of smoothing these steps and reducing the current because thermal fluctuations tend to equalize the forward and backward currents. Ohm's law is recovered for $\beta\left(\epsilon_{2}-\epsilon_{1}\right)<1$. The zerofrequency power spectrum associated to the total net current through a system interface is plotted as a function of the bias in Fig. 5(d). The Mandel parameter associated with the $\eta$ $=1$ and $\eta=2$ processes is plotted in Figs. 5(e) and 5(f). We see that the deviations from Poisson statistics are always strong and tend to vanish only when the currents associated with $\eta=1$ and $\eta=2$ vanish. This indicates that the various types of electron transfer are highly correlated with each other. The Mandel parameter is always negative, indicating sub-Poissonian (antibunching) statistics. This has been experimentally observed in Ref. 46.

\section{DISCUSSION}

Many different types of fluctuation theorems (FTs) have been derived for stochastic dynamical systems. These differ by the mechanism used to drive the system out of equilibrium. In the first case, ${ }^{2,3,9}$ the system is closed and driven by a time-dependent force which makes the rate matrix of the birth and death master equation time dependent. When the driving stops, the system will eventually reach equilibrium because the rate matrix is detail balanced. ${ }^{2}$ In the second case, ${ }^{6,8,9,20}$ the system is open and the rate matrix is not detail balanced. Even without driving, the system will eventually reach a nonequilibrium steady state. A third class of FTs (Refs. 56-58) considers the fluctuation of an entropy associated with the excess heat produced when time-dependent driving induces transitions between different nonequilibrium steady states. This paper focuses on the second case.

So far, we have assumed that the two leads of the junction have the same temperature but different chemical potentials $\mu_{L}=\mu_{0}+\Delta \mu$ and $\mu_{R}=\mu_{0}$. One could wonder what happens to the FTs if one considers different temperatures for the two leads $\beta_{L}=\beta_{0}+\Delta \beta=$ and $\beta_{R}=\beta_{0}$. In such a case, the argument of the exponential on the right-hand side of Eq. (5) becomes $x \equiv \beta_{0} \Delta \mu-\Delta \beta\left(\epsilon_{s}-\mu_{0}\right)+\Delta \beta \Delta \mu$. This implies for the orbital GF that the analog of the symmetry (27) becomes $S_{s}\left(\lambda_{(y, s)}\right)$ $=S_{s}\left(x-\lambda_{(y, s)}\right)$. However, since $x$ is different for each orbital (due to $\epsilon_{s}$ ), the many-body GF, which is given by the sum of the orbital GFs, does not possess the analog of the symmetry (29). It is therefore only for a single-orbital model that a FT $P(t, k) / P(t,-k)=\exp \{x k\}$ holds.

In summary, we have applied the quantum master equation derived in Ref. 47 for calculating the counting statistics of electrons tunneling through a quantum junction made of a system embedded between two leads. Using a generating function formalism, we derived an evolution equation for the generating operator which allows us to calculate the timedependent probability distribution of electron transfer events. This equation can be solved analytically because the manybody generating operator is a product of single-orbital generating operators. We then demonstrated that the net number of electrons crossing a given system-lead interface satisfies a FT at long times. This implies that measuring a net number of electron transfers in the direction favored by the bias is exponentially more probable than measuring it in the opposite direction. Since the argument in the exponential is the work needed to transfer the measured electrons through the junction, this fluctuation theorem can be viewed as a Crooks relation. ${ }^{2,3}$ We furthermore described how the moments of the current distribution can be deduced from the electron counting statistics and gave analytical expressions for currents and power spectra. Numerical calculations of the probability distribution for the electron counts for a model of two coupled quantum dots demonstrated that the FT becomes valid for long measurement times. Finite-time deviations were estimated.

Several future extensions of this work are called for. The first is to find if electron-electron interactions affect the FT. Another problem is to investigate if the FT still holds in a system where the population dynamics couple to the coherence dynamics (e.g., a quantum junction externally driven by a laser). In analogy to the excess heat, ${ }^{57}$ one could also consider fluctuations of excess currents, produced during transition between steady states. 

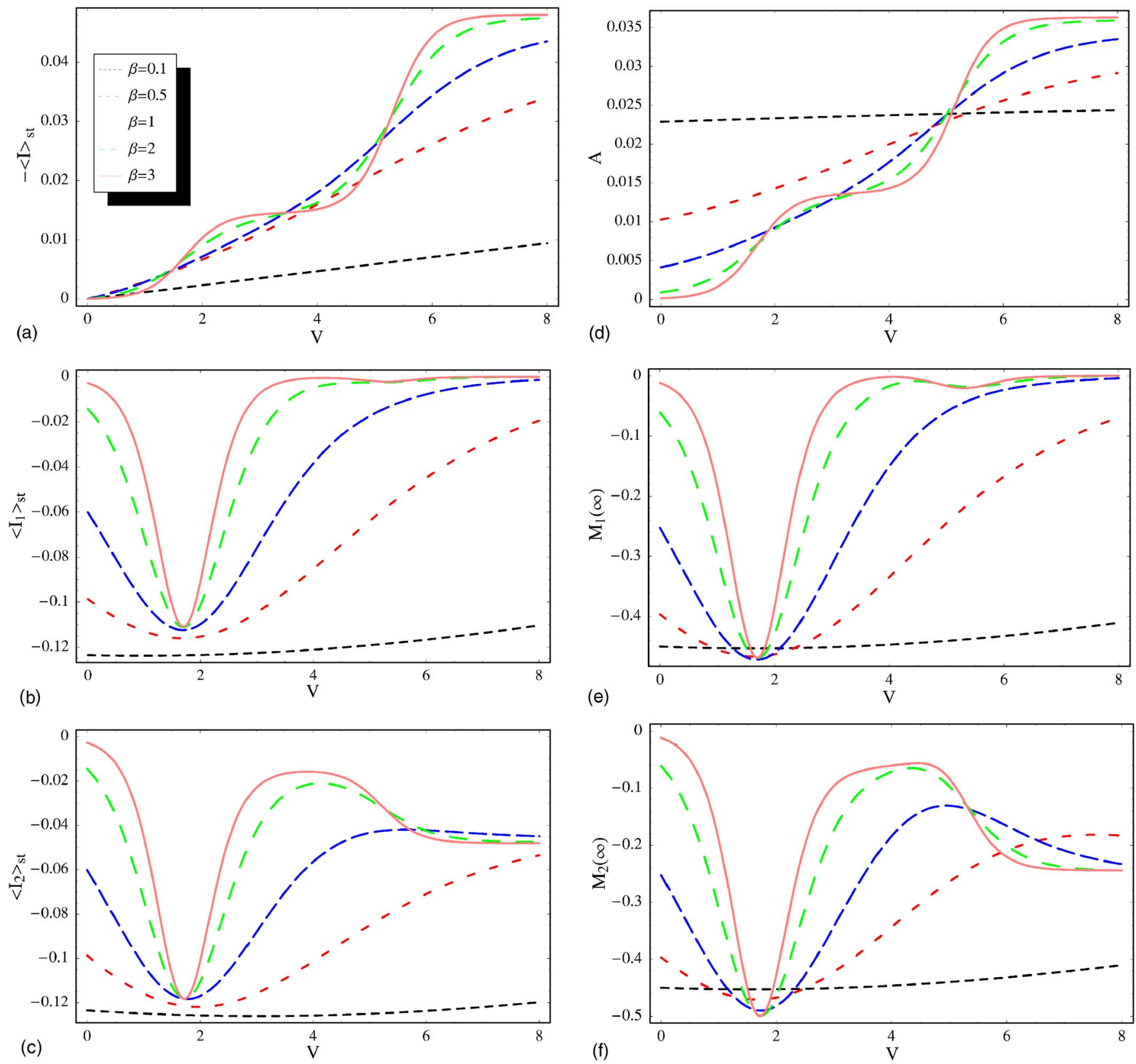

FIG. 5. (Color online) (a) Average steady-state net current $\langle I\rangle_{\mathrm{st}}$. The bias is at resonance with the dot levels for $e V=\epsilon_{1}=1.697$ and $e V$ $=\epsilon_{2}=5.303$. (b) Average current due to the electron exiting the dots from the left side of the junction (processes $\eta=1$ on Fig. 1). (c) Average current due to the electron entering the dots from the left side of the junction (processes $\eta=2$ on Fig. 1). (d) Zero-frequency power spectrum of the steady-state net current $\left[A_{1}+A_{2}\right.$ using Eq. (35)]. (e) Mandel parameter associated to the process $\eta=1$. (f) Mandel parameter associated to the process $\eta=2$. All plots are functions of the bias and are given for five different temperatures.

\section{ACKNOWLEDGMENTS}

The support of the National Science Foundation (Grant No. CHE-0446555) and NIRT (Grant No. EEC 0303389) is gratefully acknowledged. M.E. was partially supported by the FNRS Belgium (collaborateur scientifique).

\section{APPENDIX A: TRAJECTORY PICTURE FOR THE QME DYNAMICS}

Using the interaction representation, we can recast Eq. (6) as where

$$
\left.\left.\left|\dot{\rho}_{I}(t)\right\rangle\right\rangle=\hat{\Gamma}(t)\left|\rho_{I}(t)\right\rangle\right\rangle
$$

and

$$
\left.\left.\left|\rho_{I}(t)\right\rangle\right\rangle \equiv \hat{\mathcal{U}}_{0}(0, t)|\rho(t)\rangle\right\rangle
$$

$$
\hat{\Gamma}(t)=\hat{\mathcal{U}}_{0}(0, t) \hat{\Gamma} \hat{\mathcal{U}}_{0}(t, 0),
$$

where $\hat{\mathcal{U}}_{0}(0, t) \equiv \exp \left[-\hat{\mathcal{M}}_{0} t\right] . \hat{\mathcal{M}}_{0}=\hat{\mathcal{L}}+\hat{\gamma}$ describes the system dynamics in absence of electron transfer. The formal solution of Eq. (A1) reads 


$$
\left.\left.\left.\left|\rho_{I}(t)\right\rangle\right\rangle=\exp _{+}\left(\int_{0}^{t} d \tau \hat{\Gamma}(\tau)\right)\left|\rho_{I}(0)\right\rangle\right\rangle=\sum_{k=0}^{\infty}\left|\rho_{I}^{(k)}(t)\right\rangle\right\rangle,
$$

where

$$
\begin{aligned}
\left.\left|\rho_{I}^{(k)}(t)\right\rangle\right\rangle= & \int_{0}^{t} d \tau_{k} \int_{0}^{\tau_{k}} d \tau_{k-1} \cdots \int_{0}^{\tau_{2}} d \tau_{1} \hat{\Gamma}\left(\tau_{k}\right) \hat{\Gamma}\left(\tau_{k-1}\right) \cdots \\
& \left.\left.\times \hat{\Gamma}\left(\tau_{1}\right)\left|\rho_{I}(0)\right\rangle\right\rangle=\int_{0}^{t} d \tau \hat{\Gamma}(\tau)\left|\rho_{I}^{(k-1)}(\tau)\right\rangle\right\rangle .
\end{aligned}
$$

Multiplying Eq. (A5) by $\hat{\mathcal{U}}_{0}(t, 0)$, we get

$$
\begin{aligned}
\left.\left|\rho^{(k)}(t)\right\rangle\right\rangle= & \int_{0}^{t} d \tau_{k} \int_{0}^{\tau_{k}} d \tau_{k-1} \cdots \int_{0}^{\tau_{2}} d \tau_{1} \hat{\mathcal{U}}_{0}\left(t, \tau_{k}\right) \\
& \left.\times \hat{\Gamma} \hat{\mathcal{U}}_{0}\left(\tau_{k}, \tau_{k-1}\right) \hat{\Gamma} \cdots \hat{\mathcal{U}}_{0}\left(\tau_{2}, \tau_{1}\right) \hat{\Gamma} \hat{\mathcal{U}}_{0}\left(\tau_{1}, 0\right)|\rho(0)\rangle\right\rangle .
\end{aligned}
$$

This is the density matrix conditional to the transfer of $k$ electrons between the system and the leads, irrespective of the type of transfer $\eta$ or orbital $s$.

We shall denote the number of electron transfers of type $\eta$ through the $s$ orbital by $k_{\nu}$ where $\nu=(\eta, s)$. The number of electron transfers of type $\eta$ disregarding the orbital is $k_{\eta}$ $=\sum_{s=1}^{M} k_{(\eta, s)}$ and the number of electron transfer through the orbital $s$ disregarding the type of transfer is $k_{s}=\sum_{\eta=1}^{4} k_{(\eta, s)}$. We have that $k=\sum_{\nu} k_{v}$. We define

$$
\begin{gathered}
\mathbf{k}=k_{(1,1)}, k_{(1,2)}, \ldots, k_{(1, M)}, k_{(2,1)}, k_{(2,2)}, \ldots, k_{(2, M)}, \ldots, k_{(4,1)}, \\
k_{(4,2)}, \ldots, k_{(4, M)}, \\
\mathbf{k}_{s}=k_{(1, s)}, k_{(2, s)}, k_{(3, s)}, k_{(4, s)}, \\
\mathbf{k}_{\eta}=k_{(\eta, 1)}, k_{(\eta, 2)}, \ldots, k_{(\eta, M)} .
\end{gathered}
$$

A trajectory $(\nu, t)_{(\tau)}$ records (from left to right) the sequence of electron transfer events during a time $\tau$, by labeling them according to the type of transfer, the relevant orbital and the time at which a given transfer occurs $(\nu, t)_{(\tau)}$ $=\left(\nu_{1}, t_{1}\right),\left(\nu_{2}, t_{2}\right), \ldots,\left(\nu_{k}, t_{k}\right)$. A sequence $(\nu)_{(\tau)}$ is a trajectory where the transfer time is not recorded $(\nu)_{(\tau)}=\nu_{1}, \nu_{2}, \ldots, \nu_{k}$.

We can now decompose $\rho_{I}(t)$ as

$$
\left.\left.\left|\rho_{I}(t)\right\rangle\right\rangle=\sum_{\mathbf{k}}\left|\rho_{I}^{(\mathbf{k})}(t)\right\rangle\right\rangle,
$$

where the sum is over all the component $k_{\nu}$ of $\mathbf{k}$ and runs from zero to infinity. Using Eq. (12), the probability to measure $\mathbf{k}$ electrons transferred to the leads at time $t$ is given by

$$
P(t, \mathbf{k})=\left\langle\left\langle I\left|\hat{\mathcal{U}}_{0}(t, 0)\right| \rho_{I}^{(\mathbf{k})}(t)\right\rangle\right\rangle .
$$

The master equation (6) preserves the trace so that the normalization condition $\Sigma_{\mathbf{k}} P(t, \mathbf{k})=1$ is satisfied.

We next define the elementary probability density of a given trajectory $(\nu, \tau)_{(t)}$ which contains $k$ electron transfer events at time $\tau_{1}, \ldots, \tau_{k}$ during a time interval $t$ as

$$
\begin{aligned}
\Pi\left[(\nu, \tau)_{(t)}\right] \equiv & \left\langle\langle\operatorname{Tr}| \hat{\mathcal{U}}_{0}(t, 0) \hat{\Gamma}_{v_{k}}\left(\tau_{k}\right)\right. \\
& \left.\times \hat{\Gamma}_{\nu_{k-1}}\left(\tau_{k-1}\right) \ldots \hat{\Gamma}_{\nu_{1}}\left(\tau_{1}\right)\left|\rho_{I}(0)\right\rangle\right\rangle .
\end{aligned}
$$

The probability of a sequence $(\nu)_{(t)}$ with $k$ events is obtained by time integrating Eq. (A10),

$$
\Pi\left[(\nu)_{(t)}\right]=\int_{0}^{t} d \tau_{k} \int_{0}^{\tau_{k}} d \tau_{k-1} \ldots \int_{0}^{\tau_{2}} d \tau_{1} \Pi\left[(\nu, \tau)_{(t)}\right] .
$$

Using Eq. (A6), and since our equation conserves the trace, we see that $\Sigma_{(\nu)_{(t)}} \Pi\left[(\nu)_{(t)}\right]=1$, where $\Sigma_{(\nu)_{(t)}}$ is the sum over all possible electron transfer sequences (without keeping track of transfer times). The trace of Eq. (A5) can now be written using Eqs. (A10) and (A11) as

$$
P(t, \mathbf{k})=\sum_{(\nu)_{(t)} \in \mathbf{k}} \Pi\left[(\nu)_{(t)}\right],
$$

where the summation is restricted to sequences such that the number of transfer events is $\mathbf{k}$.

Because a number $\mathbf{k}$ of electrons at time $t$ can be realized by the four types of electron transfer processes (Fig. 1) and via $M$ different orbitals, we have

$$
\left.\left.\left|\rho_{I}^{(\mathbf{k})}(t)\right\rangle\right\rangle=\sum_{\nu} \int_{0}^{t} d \tau \hat{\Gamma}_{\nu}(\tau)\left|\rho_{I}^{\left(\mathbf{k}-\mathbf{1}_{\nu}\right)}(\tau)\right\rangle\right\rangle,
$$

where $\mathbf{1}_{\nu}=0, \ldots, 0,1,0, \ldots, 0$, where 1 is at the position $\nu$ in the sequence.

Using the interaction picture of the GO, we can rewrite Eq. (A13) as

$$
\left.\left.\left|G_{I}(t, \boldsymbol{\lambda})\right\rangle\right\rangle=\int_{0}^{t} d \tau\left(\sum_{\nu} e^{\lambda_{\nu}} \hat{\Gamma}_{\nu}(\tau)\right)\left|G_{I}(\tau, \boldsymbol{\lambda})\right\rangle\right\rangle .
$$

By taking the time derivative and going back to the Schrödinger picture, we get Eq. (16).

\section{APPENDIX B: SOLUTION OF THE GF}

The generator of Eq. (16) can be written as a sum of contributions of each orbital

$$
\hat{\mathcal{W}}(\boldsymbol{\lambda})=\sum_{s=1}^{M} \hat{\mathcal{W}}_{s}\left(\boldsymbol{\lambda}_{s}\right),
$$

where

$$
\hat{\mathcal{W}}_{s}\left(\boldsymbol{\lambda}_{s}\right)=\hat{\mathcal{L}}_{s}+\hat{\gamma}_{s}+\sum_{\eta=1}^{4} e^{\lambda(\eta, s)} \hat{\Gamma}_{(\eta, s)}
$$

and $\hat{\mathcal{L}}_{s} \equiv-i \epsilon_{s}\left[c_{s}^{\dagger} c_{s}, \cdot\right]$. The GO can therefore be factorized as a tensor product of orbital GO,

$$
\left.|G(t, \boldsymbol{\lambda})\rangle\rangle=\prod_{s=1}^{M}\left|G_{s}\left(t, \boldsymbol{\lambda}_{s}\right)\right\rangle\right\rangle \otimes,
$$

which evolve independently according to 


$$
\left.\left.\left|\dot{G}_{s}\left(t, \boldsymbol{\lambda}_{s}\right)\right\rangle\right\rangle=\hat{\mathcal{W}}_{s}\left(\boldsymbol{\lambda}_{s}\right)\left|G_{s}\left(t, \boldsymbol{\lambda}_{s}\right)\right\rangle\right\rangle .
$$

Projecting Eq. (B4) into the system eigenbasis and using the notation

$$
\begin{aligned}
\left\langle\left\langle n_{1} \cdots n_{M} ; n_{1}^{\prime} \cdots n_{M}^{\prime} \mid G(t, \boldsymbol{\lambda})\right\rangle\right\rangle & =\prod_{s=1}^{M}\left\langle\left\langle n_{s} ; n_{s}^{\prime} \mid G_{s}\left(t, \boldsymbol{\lambda}_{s}\right)\right\rangle\right\rangle \\
& =\prod_{s=1}^{M} G_{n_{s} ; n_{s}^{\prime}}\left(t, \boldsymbol{\lambda}_{s}\right),
\end{aligned}
$$

where

we get

$$
\left(\begin{array}{c}
\dot{G}_{1 ; 1}\left(t, \boldsymbol{\lambda}_{s}\right) \\
\dot{G}_{0 ; 0}\left(t, \boldsymbol{\lambda}_{s}\right) \\
\dot{G}_{1 ; 0}\left(t, \boldsymbol{\lambda}_{s}\right) \\
\dot{G}_{0 ; 1}\left(t, \boldsymbol{\lambda}_{s}\right)
\end{array}\right)=\hat{\mathcal{W}}\left(\boldsymbol{\lambda}_{s}\right)\left(\begin{array}{c}
G_{1 ; 1}\left(t, \boldsymbol{\lambda}_{s}\right) \\
G_{0 ; 0}\left(t, \boldsymbol{\lambda}_{s}\right) \\
G_{1 ; 0}\left(t, \boldsymbol{\lambda}_{s}\right) \\
G_{0 ; 1}\left(t, \boldsymbol{\lambda}_{s}\right)
\end{array}\right),
$$

$$
\hat{\mathcal{W}}\left(\boldsymbol{\lambda}_{s}\right)=2\left(\begin{array}{cccc}
-v_{s s} & e^{\lambda}(2, s) w_{s s}^{(L)}+e^{\lambda(4, s)} w_{s s}^{(R)} & 0 & 0 \\
e^{\lambda_{(1, s)} v_{s s}^{(L)}+e^{\lambda_{(3, s)}} v_{s s}^{(R)}} & -w_{s s} & 0 & 0 \\
0 & 0 & -i \epsilon_{s}-v_{s s}-w_{s s} & 0 \\
0 & 0 & 0 & i \epsilon_{s}-v_{s s}-w_{s s}
\end{array}\right)
$$

Since we work in the reduced Liouville space (where FSCs are neglected), only the coherences such that $\sum_{s=1}^{M} n_{s}$ $=\sum_{s=1}^{M} n_{s}^{\prime}$ are kept in Eq. (B5).

The two eigenvalues of the generator (B7) corresponding to the population dynamics are given by

$$
g_{ \pm}\left(\boldsymbol{\lambda}_{s}\right)=-\left(\frac{v_{s s}+w_{s s}}{2}\right) \pm \sqrt{\left(\frac{v_{s s}+w_{s s}}{2}\right)^{2}+f\left(\boldsymbol{\lambda}_{s}\right)},
$$

where

$$
\begin{aligned}
f\left(\boldsymbol{\lambda}_{s}\right)= & v_{s s}^{(L)}\left\{\left(e^{\left(\lambda_{(1, s)}+\lambda_{(2, s)}\right)}-1\right) w_{s s}^{(L)}+\left(e^{\left(\lambda_{(1, s)}+\lambda_{(4, s)}\right)}-1\right) w_{s s}^{(R)}\right\} \\
& +v_{s s}^{(R)}\left\{\left(e^{\left(\lambda_{(2, s)}+\lambda_{(3, s)}\right)}-1\right) w_{s s}^{(L)}+\left(e^{\left(\lambda_{(3, s)}+\lambda_{(4, s)}\right)}-1\right) w_{s s}^{(R)}\right\} .
\end{aligned}
$$

The two eigenvalues corresponding to coherences are obviously given by the two lower diagonal elements of Eq. (B7) since the populations are decoupled from coherences. The right (left) eigenvectors of the generator can be easily evaluated. The two associated to the population $\left.\left.\operatorname{read} \quad \hat{\mathcal{W}}_{s}\left(\boldsymbol{\lambda}_{s}\right)\left|g_{ \pm}\left(\boldsymbol{\lambda}_{s}\right)\right\rangle\right\rangle=g_{ \pm}\left(\boldsymbol{\lambda}_{s}\right)\left|g_{ \pm}\left(\boldsymbol{\lambda}_{s}\right)\right\rangle\right\rangle \quad\left[\left\langle\left\langle\widetilde{g}_{ \pm}\left(\boldsymbol{\lambda}_{s}\right)\right| \hat{\mathcal{W}}_{s}\left(\boldsymbol{\lambda}_{s}\right)\right.\right.$ $=\left\langle\left\langle\widetilde{g}_{ \pm}\left(\boldsymbol{\lambda}_{s}\right)\right| g_{ \pm}\left(\boldsymbol{\lambda}_{s}\right)\right]$. By tracing the solution of Eq. (B6) we get the orbital GF,

$$
\begin{aligned}
G_{s}\left(t, \boldsymbol{\lambda}_{s}\right)= & c_{+}(0) e^{g_{+}\left(\boldsymbol{\lambda}_{s}\right) t}\left[g_{1 ; 1}^{+}\left(\boldsymbol{\lambda}_{s}\right)+g_{0 ; 0}^{+}\left(\boldsymbol{\lambda}_{s}\right)\right] \\
& +c_{-}(0) e^{g_{-}\left(\boldsymbol{\lambda}_{s}\right) t}\left[g_{1 ; 1}^{-}\left(\boldsymbol{\lambda}_{s}\right)+g_{0 ; 0}^{-}\left(\boldsymbol{\lambda}_{s}\right)\right],
\end{aligned}
$$

where $\quad g_{n_{s} ; n_{s}}^{ \pm}\left(\boldsymbol{\lambda}_{s}\right)=\left\langle\left\langle n_{s} ; n_{s} \mid g_{ \pm}\left(\boldsymbol{\lambda}_{s}\right)\right\rangle\right\rangle \quad$ and $\quad c_{ \pm}(0)$ $=\left\langle\left\langle\widetilde{g}_{ \pm}\left(\boldsymbol{\lambda}_{s}\right) \mid G_{s}\left(0, \boldsymbol{\lambda}_{s}\right)\right\rangle\right\rangle$. The many-body GF (18) is given by

$$
G(t, \boldsymbol{\lambda})=\prod_{s=1}^{M} G_{s}\left(t, \boldsymbol{\lambda}_{s}\right) .
$$

This constitutes the solution of Eq. (16). If one does the spectral decomposition directly on the many-body generator one gets

$$
G(t, \boldsymbol{\lambda})=\sum_{m}^{2^{M}} e^{g_{m}(\boldsymbol{\lambda}) t}\left\langle\left\langle I \mid g_{m}(\boldsymbol{\lambda})\right\rangle\right\rangle\left\langle\left\langle\widetilde{g}_{m}(\boldsymbol{\lambda}) \mid \rho(0)\right\rangle\right\rangle,
$$

where $\left.g_{m}(\boldsymbol{\lambda}),\left|g_{m}(\boldsymbol{\lambda})\right\rangle\right\rangle$ and $\left\langle\left\langle\widetilde{g}_{m}(\boldsymbol{\lambda})\right|\right.$ are, respectively, the many-body eigenvalues, right, and left eigenvector of the generator. Each of these many-body eigenvalue is made from one of the $2^{M}$ possible ways of summing the orbital eigenvalues (B8) and the many-body left and right eigenvectors are tensor products of the single-orbital eigenvectors.

\section{APPENDIX C: FLUCTUATION THEOREM DERIVED FROM THE GENERATING FUNCTION SYMMETRY}

The following reasoning is based on the steady state FT for the entropy first obtained ${ }^{6,8}$ and later extended for currents. $^{54,55}$ The GF is associated with the probability distribution by

$$
G(t, \lambda)=\sum_{k} P(t, k) e^{-\lambda k}=\int d \xi \tilde{P}(t, \xi) e^{-\lambda \xi \xi},
$$

where we have introduced $\widetilde{P}(t, \xi)$, the probability that $\xi$ $=k / t$ takes a value in the interval $[\xi, \xi+d \xi]$. The large deviation function (LDF) is defined as

$$
R(\xi) \equiv-\lim _{t \rightarrow \infty} \frac{1}{t} \ln \tilde{P}(t, \xi) .
$$

This definition follows from the ansatz

$$
\widetilde{P}(t, \xi)=C(\xi, t) e^{-R(\xi) t},
$$

where 


$$
\lim _{t \rightarrow \infty} \frac{1}{t} \ln C(\xi, t)=0 .
$$

We can then rewrite Eq. (C1) as

$$
G(t, \lambda)=\int d \xi C(\xi, t) e^{-(R(\xi)+\lambda \xi) t} .
$$

At long times, the main contribution to this integral comes from the value of $\xi, \xi^{*}$, that maximizes the argument of the exponential. $\xi^{*}$ is therefore the value of $\xi$ such that $\lambda$ $=-\left.\frac{d R}{d \xi}\right|_{\xi=\xi^{*}}$. At long times, using steepest descent integration, Eq. (C5) becomes

$$
\begin{aligned}
G(t, \lambda) & \left.\left.\approx e^{-\left(R\left(\xi^{*}\right)+\lambda \xi^{*}\right) t} \int d \xi C(\xi, t) e^{-(1 / 2)\left[\left(d^{2} R(\xi) / d \xi^{2}\right)\right.}\right|_{\xi=\xi^{*}}\right]\left(\xi-\xi^{*}\right)^{2} t \\
& \approx e^{-\left[R\left(\xi^{*}\right)+\lambda \xi^{*}\right] t} C\left(\xi^{*}, t\right)\left[\left(\left.\frac{d^{2} R(\xi)}{d \xi^{2}}\right|_{\xi=\xi^{*}}\right) \frac{t}{2 \pi}\right]^{-1 / 2} .
\end{aligned}
$$

Substituting Eq. (C6) in Eq. (21) gives

$$
S(\lambda)=R\left(\xi^{*}\right)+\lambda \xi^{*} .
$$

This shows that $S(\lambda)$ is the Legendre transform of the LDF. The LDF is given by the inverse Legendre transform of $S(\lambda)$,

$$
R(\xi)=S\left(\lambda^{*}\right)-\lambda^{*} \xi
$$

where $\xi=\left.\frac{d S}{d \lambda}\right|_{\lambda=\lambda^{*}}$. Since $S(\lambda)$ is convex downward [i.e., $\left.d^{2} S / d \lambda^{2}=-\lim _{t \rightarrow \infty}\left(\left\langle k^{2}\right\rangle_{t}-\langle k\rangle_{t}^{2}\right) \leq 0\right]$, its Legendre transform is convex upwards. Using the symmetry (29), Eq. (C8) implies that $R(-\xi)=S(\beta e V-\lambda)+(\beta e V-\lambda) \xi$, which together with $R(\xi)=S(\lambda)-\lambda \xi$ leads to

$$
R(\xi)-R(-\xi)=-\beta e V \xi .
$$

Substituting this in Eq. (C3), we get

$$
\ln \frac{\widetilde{P}(t, \xi)}{\widetilde{P}(t,-\xi)}=\beta e V \xi t+\ln \frac{C(\xi, t)}{C(-\xi, t)} .
$$

Using Eq. (C4), this gives the FT (30) in the long time limit.

\section{APPENDIX D: CURRENT, POWER SPECTRUM, AND THE GENERATING FUNCTION}

We show how to calculate the average currents, their zero-frequency power spectra, and their Mandel parameter from the GF. These results are used in Sec. V.

To simplify the notation, we assume we have a probability distribution $P(t, \mathbf{k})$ and its associated generating function $G(t, \boldsymbol{\lambda})=\Sigma_{\mathbf{k}} P(t, \mathbf{k}) e^{\boldsymbol{\lambda} \cdot \mathbf{k}}$, where the component of $\mathbf{k}$ and $\boldsymbol{\lambda}$ are given by $k_{\eta}$ and $\lambda_{\eta}$. We also have $S(\boldsymbol{\lambda})$ $=-\lim _{t \rightarrow \infty} \frac{1}{t} \ln G(t, \boldsymbol{\lambda})$. The averaged number of charge $\eta$ is given by

$$
\left\langle k_{\eta}\right\rangle_{t}=-\frac{1}{e} \int_{0}^{t} d \tau\left\langle I_{\eta}(\tau)\right\rangle=\left.\frac{\partial}{\partial \lambda_{\eta}} G(t, \boldsymbol{\lambda})\right|_{\lambda=0},
$$

and the steady-state current by

$$
\left\langle I_{\eta}\right\rangle_{\mathrm{st}}=\lim _{t \rightarrow \infty} \frac{1}{t} \int_{0}^{t} d \tau\left\langle I_{\eta}(\tau)\right\rangle=\left.e \frac{\partial}{\partial \lambda_{\eta}} S(\boldsymbol{\lambda})\right|_{\boldsymbol{\lambda}=0} .
$$

We also find that

$$
\begin{aligned}
\left\langle k_{\eta} k_{\eta^{\prime}}\right\rangle_{t} & =\left.\frac{\partial}{\partial \lambda_{\eta}} \frac{\partial}{\partial \lambda_{\eta^{\prime}}} G(t, \boldsymbol{\lambda})\right|_{\lambda=0} \\
& =\frac{1}{e^{2}} \int_{0}^{t} d \tau_{1} \int_{0}^{t} d \tau_{2}\left\langle I_{\eta}\left(\tau_{1}\right) I_{\eta^{\prime}}\left(\tau_{2}\right)\right\rangle .
\end{aligned}
$$

Since at steady state $\left\langle I_{\eta}\left(\tau_{1}\right) I_{\eta^{\prime}}\left(\tau_{2}\right)\right\rangle_{s t}=\left\langle I_{\eta}\left(\tau_{1}-\tau_{2}\right) I_{\eta^{\prime}}(0)\right\rangle_{s t}$, $\left\langle I_{\eta}\left(\tau_{1}\right)\right\rangle_{s t}=\left\langle I_{\eta}\right\rangle_{s t}$, and $\left\langle I_{\eta^{\prime}}\left(\tau_{2}\right)\right\rangle_{s t}=\left\langle I_{\eta^{\prime}}\right\rangle_{s t}$, using the fact that

$$
\left.\frac{\partial}{\partial \lambda_{\eta}} \frac{\partial}{\partial \lambda_{\eta^{\prime}}} \ln G(t, \boldsymbol{\lambda})\right|_{\boldsymbol{\lambda}=0}=\left\langle k_{\eta} k_{\eta^{\prime}}\right\rangle_{t}-\left\langle k_{\eta}\right\rangle_{t}\left\langle k_{\eta^{\prime}}\right\rangle_{t}
$$

we find

$$
\begin{aligned}
A_{\eta \eta^{\prime}} & =\left.e^{2} \frac{\partial}{\partial \lambda_{\eta}} \frac{\partial}{\partial \lambda_{\eta^{\prime}}} S(\boldsymbol{\lambda})\right|_{\lambda=0} \\
& =\int_{-\infty}^{\infty} d \tau\left(\left\langle I_{\eta}(\tau) I_{\eta^{\prime}}(0)\right\rangle_{s t}-\left\langle I_{\eta}\right\rangle_{s t}\left\langle I_{\eta^{\prime}}\right\rangle_{s t}\right) \\
& =\int_{-\infty}^{\infty} d \tau\left\langle\left[I_{\eta}(\tau)-\left\langle I_{\eta}\right\rangle_{s t}\right]\left[I_{\eta^{\prime}}(0)-\left\langle I_{\eta^{\prime}}\right\rangle_{s t}\right]\right\rangle_{s t} .
\end{aligned}
$$

Since the Fourier transform of the current correlation function is given by

$$
A_{\eta \eta^{\prime}}(\omega)=\int_{-\infty}^{\infty} d \tau e^{-i \omega \tau}\left\langle\left[I_{\eta}(\tau)-\left\langle I_{\eta}\right\rangle_{s t}\right]\left[I_{\eta^{\prime}}(0)-\left\langle I_{\eta^{\prime}}\right\rangle_{s t}\right]\right\rangle_{s t},
$$

we see that $A_{\eta \eta^{\prime}}$ in Eq. (D5) is the zero-frequency power spectrum of the current correlation function $A_{\eta \eta^{\prime}}=A_{\eta \eta^{\prime}}(\omega$ $=0$ ). This quantity is used to study shot noise. $\cdot^{32}$

The analog of the Mandel parameter in photon counting statistic $^{28,29}$ for the process $y$ is given by

$$
M_{\eta}(t) \equiv \frac{\left(\left\langle k_{\eta}^{2}\right\rangle_{t}-\left\langle k_{\eta}\right\rangle_{t}^{2}\right)-\left\langle k_{\eta}\right\rangle_{t}}{\left\langle k_{\eta}\right\rangle_{t}}=\frac{\left.\left(\partial^{2} / \partial \lambda_{\eta}^{2}\right) \ln G(t, \boldsymbol{\lambda})\right|_{\lambda=0}}{\left.\left(\partial / \partial \lambda_{\eta}\right) G(t, \boldsymbol{\lambda})\right|_{\lambda=0}}-1 .
$$

The asymptotic value is given by

$$
M_{\eta}(\infty)=\frac{\left.\left(\partial^{2} / \partial \lambda_{\eta}^{2}\right) S(\boldsymbol{\lambda})\right|_{\lambda=0}}{\left.\left(\partial / \partial \lambda_{\eta}\right) S(\boldsymbol{\lambda})\right|_{\lambda=0}}-1 .
$$

For a Poisson process $M_{\eta}=0$. The zero-frequency power spectrum is related to the long time limit of the Mandel parameter by

$$
A_{\eta \eta}=e\left\langle I_{\eta}\right\rangle_{\mathrm{st}}\left[1+M_{\eta}(\infty)\right] .
$$

\section{APPENDIX E: GENERATOR FOR THE TWO QUANTUM DOT MODEL}

We present the basic quantities needed in the reduced 
Liouville space to study the model of two quantum dot model presented in Sec. IV. The orbital eigenbasis is denoted by $\left\{\left|n_{1}, n_{2}\right\rangle\right\}$, where $n_{1}\left(n_{2}\right)$ is the occupation number of the orbital $s=1 \quad(s=2)$. Using the notation $\left\langle n_{1}, n_{2}|\rho| n_{1}^{\prime}, n_{2}^{\prime}\right\rangle$ $=\rho_{n_{1} n_{2} ; n_{1}^{\prime} n_{2}^{\prime}}$, the density matrix in the reduced Liouville space is given by the vector $\rho=\left(\rho_{00 ; 00}, \rho_{01 ; 01}, \rho_{10 ; 10}, \rho_{11 ; 11}\right.$, $\left.\rho_{10 ; 01}, \rho_{01 ; 10}\right)^{T}$. The generator of our QME (1) for this model in this basis reads

$$
\hat{\mathcal{M}}=\left(\begin{array}{cccccc}
-2\left(w_{11}+w_{22}\right) & 2 v_{22} & 2 v_{11} & 0 & 0 & 0 \\
2 w_{22} & -2\left(v_{22}+w_{11}\right) & 0 & 2 v_{11} & 0 & 0 \\
2 w_{11} & 0 & -2\left(v_{11}+w_{22}\right) & 2 v_{22} & 0 & 0 \\
0 & 2 w_{11} & 2 w_{22} & -2\left(v_{11}+v_{22}\right) & 0 & 0 \\
0 & 0 & 0 & 0 & -\mathcal{X} & 0 \\
0 & 0 & 0 & 0 & 0 & -\mathcal{X}^{*},
\end{array}\right),
$$

where $\mathcal{X}=v_{11}+w_{11}+v_{22}+w_{22}+i\left(\epsilon_{1}-\epsilon_{2}\right)$. As expected, the populations are decoupled from the coherences. The generator is diagonal for the coherences and obeys a birth and death master equation in the population space. The generator for the GO evolution equation (16) is given by

$$
\hat{\mathcal{W}}(\boldsymbol{\lambda})=\left(\begin{array}{cccc}
-2\left(w_{11}+w_{22}\right) & 2\left(e^{\lambda_{(1,2)}} v_{22}^{(L)}+e^{\lambda_{(3,2)}} v_{22}^{(R)}\right) & 2\left(e^{\left.\lambda_{(1,1}\right)} v_{11}^{(L)}+e^{\lambda_{(3,1}} v_{11}^{(R)}\right) & 0 \\
2\left(e^{\lambda_{(2,2)}} w_{22}^{(L)}+e^{\lambda(4,2)} w_{22}^{(R)}\right) & -2\left(v_{22}+w_{11}\right) & 0 & 2\left(e^{\lambda_{(1,1)}} v_{11}^{(L)}+e^{\lambda_{(3,1)}} v_{11}^{(R)}\right) \\
2\left(e^{\lambda_{(2,1)}} w_{11}^{(L)}+e^{\lambda(4,1)} w_{11}^{(R)}\right) & 0 & -2\left(v_{11}+w_{22}\right) & 2\left(e^{\lambda_{(1,2)}} v_{22}^{(L)}+e^{\lambda_{(3,2)}} v_{22}^{(L)}\right) \\
0 & 2\left(e^{\lambda_{(2,1)}} w_{11}^{(L)}+e^{\lambda_{(4,1)}} w_{11}^{(R)}\right) & 2\left(e^{\lambda_{(2,2)}} w_{22}^{(L)}+e^{\lambda_{(4,2)}} w_{22}^{(L)}\right) & -2\left(v_{11}+v_{22}\right)
\end{array}\right),
$$

where the coherence part has been discarded since it is the same as for the generator of the QME.

*Also at Center for Nonlinear Phenomena and Complex Systems, Universite Libre de Bruxelles, Code Postal 231, Campus Plaine, B-1050 Brussels, Belgium.

${ }^{1}$ C. Jarzynski, Phys. Rev. Lett. 78, 2690 (1997); Phys. Rev. E 56, 5018 (1997).

${ }^{2}$ G. E. Crooks, Phys. Rev. E 60, 2721 (1999).

${ }^{3}$ G. E. Crooks, Phys. Rev. E 61, 2361 (2000).

${ }^{4}$ G. Gallavotti and E. G. D. Cohen, Phys. Rev. Lett. 74, 2694 (1995).

${ }^{5}$ J. Kurchan, J. Phys. A 31, 3719 (1998).

${ }^{6}$ J. L. Lebowitz and H. Spohn, J. Stat. Phys. 95, 333 (1999).

${ }^{7}$ D. J. Searles and D. J. Evans, Phys. Rev. E 60, 159 (1999).

${ }^{8}$ P. Gaspard, J. Chem. Phys. 120, 8898 (2004).

${ }^{9}$ U. Seifert, Phys. Rev. Lett. 95, 040602 (2005).

${ }^{10}$ B. Cleuren, C. Van den Broeck, and R. Kawai, Phys. Rev. E 74, 021117 (2006).

${ }^{11}$ C. Maes, Séminaire Poincaré 2, 29 (2003).

${ }^{12}$ P. Gaspard, Physica A 369, 201 (2006).

${ }^{13}$ C. Bustamante, J. Liphardt, and F. Ritort, Phys. Today 58(7), 43 (2005).

${ }^{14}$ D. Collin, F. Ritort, C. Jarzynski, S. B. Smith, I. Tinoco, Jr., and C. Bustamante, Nature (London) 437, 231 (2005).

${ }^{15}$ S. Mukamel, Phys. Rev. Lett. 90, 170604 (2003).

${ }^{16}$ T. Monnai and S. Tasaki, cond-mat/0308337 (unpublished).

${ }^{17}$ W. De Roeck and C. Maes, Phys. Rev. E 69, 026115 (2004).

${ }^{18}$ T. Monnai, Phys. Rev. E 72, 027102 (2005).

${ }^{19}$ A. E. Allahverdyan and Th. M. Nieuwenhuizen, Phys. Rev. E 71, 066102 (2005).
${ }^{20}$ W. De Roeck and C. Maes, Rev. Math. Phys. 18, 619 (2006).

${ }^{21}$ M. Esposito and S. Mukamel, Phys. Rev. E 73, 046129 (2006).

${ }^{22}$ R. J. Glauber, Phys. Rev. 131, 2766 (1963).

${ }^{23}$ P. L. Kelley and W. H. Kleiner, Phys. Rev. 136, A316 (1964).

${ }^{24}$ L. Mandel, Phys. Rev. Lett. 49, 136 (1982).

${ }^{25}$ Y. Zheng and F. L. H. Brown, Phys. Rev. Lett. 90, 238305 (2003); J. Chem. Phys. 119, 11814 (2003).

${ }^{26}$ S. Mukamel, Phys. Rev. A 68, 063821 (2003).

${ }^{27}$ F. Sanda and S. Mukamel, Phys. Rev. A 71, 033807 (2005).

${ }^{28}$ F. Sanda and S. Mukamel, J. Chem. Phys. 124, 124103 (2006).

${ }^{29}$ E. Barkai, Y. Jung, and R. Silbey, Annu. Rev. Phys. Chem. 55, 457 (2004).

${ }^{30}$ F. Kulzer and M. Orrit, Annu. Rev. Phys. Chem. 55, 585 (2004).

${ }^{31}$ L. S. Levitov and M. Reznikov, Phys. Rev. B 70, 115305 (2004); L. S. Levitov and M. Reznikov, cond-mat/0111057 (unpublished).

${ }^{32}$ Y. M. Blanter and M. Buttiker, Phys. Rep. 336, 1 (2000).

${ }^{33}$ S. A. Gurvitz, Phys. Rev. B 56, 15215 (1997).

${ }^{34}$ J. Wabnig, D. V. Khomitsky, J. Rammer, and A. L. Shelankov, Phys. Rev. B 72, 165347 (2005).

${ }^{35}$ J. Rammer, A. L. Shelankov, and J. Wabnig, Phys. Rev. B 70, 115327 (2004).

${ }^{36}$ A. L. Shelankov and J. Rammer, Europhys. Lett. 63, 485 (2003).

${ }^{37}$ C. Flindt, T. Novotny, and A.-P. Jauho, Europhys. Lett. 69, 475 (2005).

${ }^{38}$ G. Kiesslich, P. Samuelsson, A. Wacker, and E. Schöll, Phys. Rev. B 73, 033312 (2006). 
${ }^{39}$ Y. Utsumi, D. S. Golubev, and G. Schön, Phys. Rev. Lett. 96, 086803 (2006).

${ }^{40}$ J. N. Pedersen and A. Wacker, Phys. Rev. B 72, 195330 (2005).

${ }^{41}$ D. A. Bagrets and Yu. V. Nazarov, Phys. Rev. B 67, 085316 (2003).

${ }^{42}$ W. Lu, Z. Ji, L. Pfeiffer, K. W. West, and A. J. Rimberg, Nature (London) 423, 422 (2003).

${ }^{43}$ T. Fujisawa, T. Hayashi, Y. Hirayama, and H. D. Cheong, Appl. Phys. Lett. 84, 2343 (2004).

${ }^{44}$ J. Bylander, T. Duty, and P. Delsing, Nature (London) 434, 361 (2005).

${ }^{45}$ S. Gustavsson, R. Leturcq, B. Simovic, R. Schleser, T. Ihn, P. Studerus, K. Ensslin, D. C. Driscoll, and A. C. Gossard, Phys. Rev. Lett. 96, 076605 (2006).

${ }^{46}$ T. Fujisawa, T. Hayashi, R. Tomita, and Y. Hirayama, Science 312, 1634 (2006).

${ }^{47}$ U. Harbola, M. Esposito, and S. Mukamel, Phys. Rev. B 74, 235309 (2006).

${ }^{48}$ H.-P. Breuer and F. Petruccione, The Theory of Open Quantum
Systems (Oxford University Press, Oxford, 2002).

${ }^{49}$ C. W. Gardiner and P. Zoller, Quantum Noise (Springer, Berlin, 2000).

${ }^{50}$ F. Haake, Statistical Treatment of Open Systems, Springer Tracts in Modern Physics, Vol. 66 (1973).

${ }^{51}$ H. Spohn, Rev. Mod. Phys. 53, 569 (1980).

${ }^{52}$ C. Cohen-Tannoudji, J. Dupont-Roc, and G. Grynberg, AtomPhoton interactions: Basics Processes and Applications (John Wiley and Sons, Inc., New York, 1992).

${ }^{53}$ D. Andrieux and P. Gaspard, J. Stat. Mech.: Theory Exp. (2006), P01011.

${ }^{54}$ D. Andrieux and P. Gaspard, J. Chem. Phys. 121, 6167 (2004).

${ }^{55}$ D. Andrieux and P. Gaspard, cond-mat/0512254 (unpublished).

${ }^{56}$ T. Hatano, Phys. Rev. E 60, R5017 (1999).

${ }^{57}$ T. Hatano and S. I. Sasa, Phys. Rev. Lett. 86, 3463 (2001).

${ }^{58}$ E. H. Trepagnier, C. Jarzynski, F. Ritort, G. E. Crooks, C. J. Bustamante, and J. Liphardt, Proc. Natl. Acad. Sci. U.S.A. 101, 15038 (2004). 